\title{
The Array for Nuclear Astrophysics Studies with Exotic Nuclei (ANASEN)*
}

\author{
M. Matoš, J.C. Blackmon, L.E. Linhardt, L.L. Mondello, \\ Louisiana State University \\ Dept. of Physics and Astronomy, Baton Rouge, LA 70803 \\ E-mail: matos@lsu.edu
}

\section{E. Johnson, G. Rogachev, I. Wiedenhover}

Florida State University

Physics Department, Tallahassee, FL 32306

\begin{abstract}
Reactions involving radioactive nuclei play an important role in stellar explosions, but experimental information about most reactions involving short-lived nuclei is limited. New facilities aim to provide wider access to unstable isotopes, but the limited intensities that are available require more efficient and selective techniques and devices to study the reactions important for astrophysics. The Array for Nuclear Astrophysics Studies with Exotic Nuclei (ANASEN) is a charged-particle detector array designed primarily for studies of reactions important in the $\alpha$ - and rp- processes with proton-rich exotic nuclei. The array consists of 40 silicon-strip detectors backed with CsI scintillators. The detectors cover an area of about $1300 \mathrm{~cm}^{2}$ providing essentially complete solid angle coverage for the reactions of interest with good energy and position resolution. ANASEN also includes a position-sensitive annular gas proportional counter that allows it to be used as an active gas target/detector. One of the primary goals of ANASEN is the direct measurement of $(\alpha, p)$ reactions in inverse kinematics. ANASEN is also well suited for studies of proton elastic and inelastic scattering, $(p, \alpha)$ reactions and transfer reactions, which will also be studied to indirectly determine reaction rates important for astrophysics. The array is being developed by Louisiana State University and Florida State University, and will be used primarily at the RESOLUT radioactive ion beam facility at FSU and the ReA3 facility at the National Superconducting Cyclotron Laboratory.
\end{abstract}

*Supported by the U.S. National Science Foundation and the U.S. Department of Energy.

11th Symposium on Nuclei in the Cosmos - NIC XI

Heidelberg, Germany

July 19-232010 


\section{Motivation}

The Array for Nuclear Astrophysics Studies with Exotic Nuclei (ANASEN) is being developed to study reactions using beams of short-lived radioactive ions that are important in stellar explosions like novae and X-ray bursts. ANASEN is a charged-particle detector array that will be used for direct measurements of $(\alpha, p)$ reactions and studies of nuclear structure properties through scattering and transfer reactions to improve our understanding of other reactions with radioactive ion beams important for nuclear structure and astrophysics.

\section{ANASEN Overview}

ANASEN achieves efficient detection of charged particles over a large angular range $\left(\theta_{\text {lab }} \sim 10^{\circ}-160^{\circ}\right)$ with good position and energy resolution by using three different types of detectors: silicon-strip detectors, CsI detectors, and a position-sensitive proportional counter. A total of 36 "Super X3" silicon-strip detectors are arranged in 3 rings of 12 detectors in a barrel design with a diameter of about $18 \mathrm{~cm}$ surrounding the beam axis. Each Super X3 detector is backed by a trapezoidal-shaped $\mathrm{CsI}(\mathrm{Tl})$ scintillator to detect particles that are not stopped by the silicon. ANASEN accommodates a solid target between two of the rings, or ANASEN can be used with an extended gas target by filling the chamber with gas. In the gas target/detector mode, a proportional counter, using 32 resistive $\mathrm{HV}$ anode wires located $3 \mathrm{~cm}$ from the beam axis, tags the position at which reaction products leave the beam axis. By accurately measuring the position near the beam axis and with the outer silicon detectors, the vertex of the reaction and the angle can be accurately reconstructed. A new design of annular silicon detector is also being developed for ANASEN that can enclose the most forward/backward angles to maximize solid angle coverage. A schematic illustrating one of the Super X3 and CsI rings surrounding the wires of the proportional counter is shown in Fig. 1.

\section{Development and testing}

The Super X3 silicon-strip detectors $(74.8 \mathrm{~mm} \times 40.3 \mathrm{~mm} \times 1 \mathrm{~mm})$ are based upon the $\mathrm{X} 3$ design developed originally for the Oak Ridge-Rutgers Univ. Barrel Array (ORRUBA) [1]. An illustration of the Super X3 detector is shown in the left panel of Fig. 2. The detector is divided into 4 resistive strips ( $75 \mathrm{~mm}$ long). In the Super $\mathrm{X} 3$ design, additional mask sets were added to segment the rear (ohmic) face of the detector into four $18.6 \mathrm{~mm} \times 40.3 \mathrm{~mm}$ (non-resistive) segments oriented perpendicularly to the resistive strips on the front face of the detector. This results in several significant performance improvements. First, the energy for any event is determined in the Super X3 design from the rear (non-resistive) segments. A representative energy spectrum from

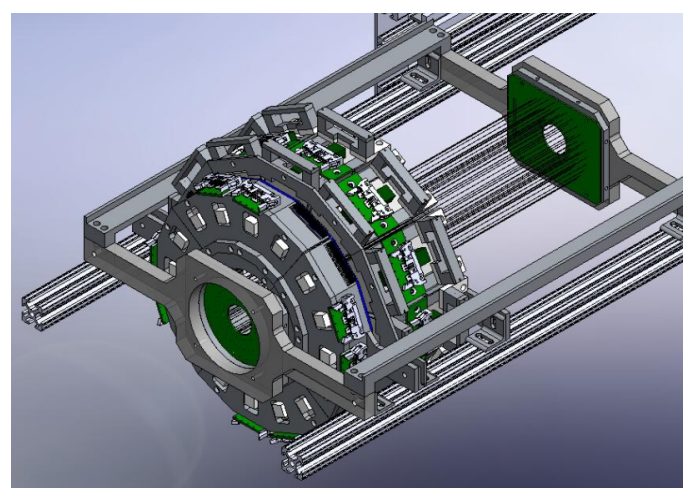

Figure 1. Drawing showing 1 of the 3 $\mathrm{Si}+\mathrm{CsI}$ rings and wires of the gas counter. 

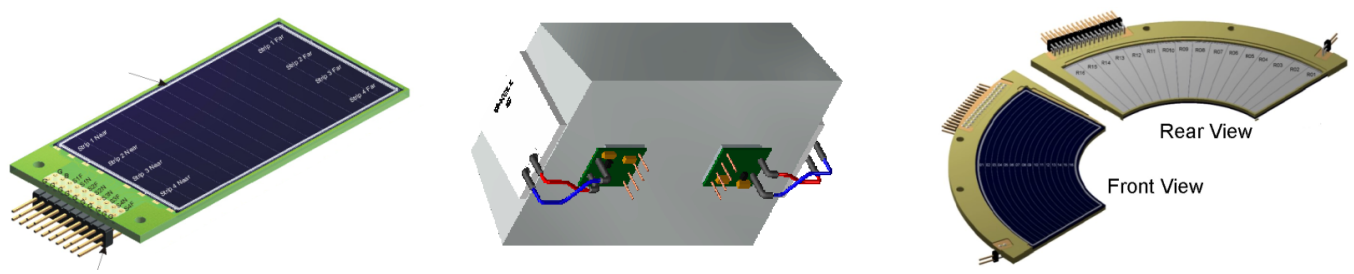

Figure 2. Left: Super X3 Si detector. Center: CSI(Tl) detector. Right: QQQ3 detector.

one of the back segments with a ${ }^{241} \mathrm{Am}$ source is shown in the left panel of Fig. 3. The measured energy resolution for the Super X3 is typically about $30 \mathrm{keV}$ in FWHM, and three alpha energies of 5388,5443 and $5486 \mathrm{keV}$ in ${ }^{241} \mathrm{Am}$ are nearly resolved. In the original X3 design the high capacitance of the common back plane resulted in relatively poor energy resolution, requiring the energy to be determined from the sum energy from the front resistive strips with a resulting resolution of about $60 \mathrm{keV}$ at best.

Achieving a precise full energy from the back face of the detector also allows the position to be determined by comparing the largest signal from the front resistive strip to the full energy from the rear face. This results in a reliable position determination over the full range of the strip without sensitivity to the electronic threshold, while in the X3 design the length over which the position is determined depends upon the energy deposited in the detector. Also shown in Fig. 3 (middle panel) is a plot of counts vs. position with a mask placed in front of the detector to restrict the position of the alphas. We find typical position resolution of about $1.5 \mathrm{~mm}$, about a factor of 2-3 worse than measured for the X3 detectors. While the origin of the poorer position resolution is not yet understood (though likely originating from the electronics) the measured resolution is comparable to beam spot size and position resolution of the proportional counter and should not adversely affect the overall position resolution.

A new annular silicon strip detector, the QQQ3, is also under development for ANASEN. The QQQ3 is designed to cover the largest possible solid angle using four silicon detectors. It has an outer active radius of $99 \mathrm{~mm}$. The inner active radius of $50 \mathrm{~mm}$ is designed to accommodate operations of the QQQ3 with the gas proportional counter. Each quadrant of the QQQ3 is segmented into 16 radial segments (3 mm pitch) and 16 angular segments. When used with a solid target, ANASEN can be used with a S2 or "CD" style annular silicon strip detector downstream of the QQQ3 to provide annular coverage to more forward angles.

In a few cases, the $1 \mathrm{~mm}$ thick silicon strip detectors are not sufficiently thick to stop protons emitted at the highest bombarding energies, so each Super X3 detector is backed with a 26mm-thick trapezoidal CsI scintillator (middle panel of Fig. 2). Each scintillator is read out by two $18 \times 18 \mathrm{~mm}^{2}$ pin-diodes. The performance of the first crystals has been tested by directly illuminating the crystals with a collimated alpha source, and a representative energy spectrum is shown in the right panel of Fig. 3. We find a typical energy resolution of about $10 \%$ that is limited by electronic noise and position dependence of the light yield. While this resolution is somewhat worse than the design resolution of about $7 \%$, it is sufficient for expected applications, and improvements in the electronics are now being implemented that are targeting a reduction in noise and an improvement in the energy resolution. The CsI detectors used with ANASEN also have good efficiency for low energy gamma rays and can be used to help distinguish reactions that populate low lying excited states in the final nucleus. 
An important feature of ANASEN is its active gas target/detector mode in which an extended helium or isobutane gas target is used to allow a broad range of center-of-mass energies to be simultaneously measured with a single bombarding energy. In this configuration the beam will enter the target chamber through a thin window. An annular proportional counter surrounds the beam axis using 32 resistive anode wires that run parallel to the beam at a distance of $3 \mathrm{~cm}$ from the beam axis. The position is determined from the relative charge measured from the ends of anode wires. The anode wires are surrounded by grounded cathode planes formed from $50 \mu \mathrm{m}$ diameter gold-plated tungsten wires at a radius of about 2 and $4 \mathrm{~cm}$ that shield the anode wires from the high ionization region near the beam axis and define a charge collection region that is about $2 \mathrm{~cm}$ thick. Anode wires have been developed using $7 \mu \mathrm{m}$ diameter carbon fiber wires (resistance $\sim 4 \mathrm{k} \Omega / \mathrm{cm}$ ) that have 5 times better tensile strength than typical metal alloy. The thin diameter wire allows high gain to be achieved at modest voltages, which is needed to observe small signals from high energy protons at the relatively modest gas pressures that are required to keep the energy loss of the incident beam per unit length acceptably low to achieve good center-of-mass resolution from the vertex reconstruction. However, a broader dynamic range must be covered to encompass both high-energy protons (small energy loss) and recoiling heavy ions (high energy loss). A prototype 16-channel preamplifier produced by Mesytec have been tested and will be used for this purpose. The gaseous target is expected to handle intensities at least up to $10^{6} \mathrm{pps}$ and provide position of the reaction vertex determined with resolution of about $3 \mathrm{~mm}$.

More than 700 individual channels of electronics will be employed in ANASEN. To minimize cost, we are collaborating with Washington University to implement a data acquisition system common to all the detector systems based around a new version of HINP16C Application Specific Integrated Circuit [2] originally developed for the High Resolution Array (HiRA) [3]. External preamplifiers are being developed for each type of detector to optimally match the signals from each detector to the gain and time constant of the ASIC shapers. A 72channel preamplifier motherboard has been developed based upon the preamplifier chips developed for the LASSA array [4]. Logarithmic HV preamplifiers developed by Mesytec will be utilized for the proportional counter, and high-gain preamplifiers are being incorporated with each diode readout for the CsI. Testing of the ASIC system with silicon-strip detectors and sources has been completed, and we are next working on testing the data acquisition system with the CsI and proportional counter prototypes.
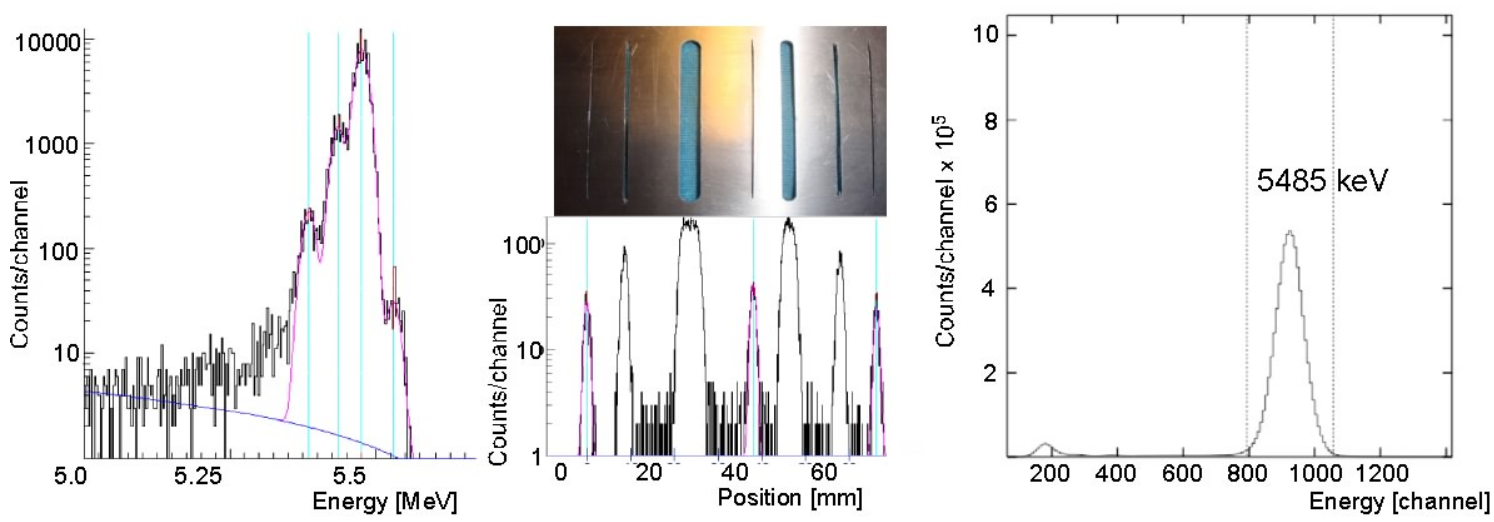

Figure 3. Left: Energy resolution measurement of the non-resistive side of the Super-X3. Center: Photograph of a mask placed in front of a Super-X3 compared to the meaured spectrum of counts vs. position. Right: Energy spectrum from the CsI prototype using a collimated ${ }^{241}$ Am alpha source. 


\section{Monte Carlo Simulation}

A GEANT4 Monte Carlo simulation has been developed to study the performance of the detector for various reactions and experimental conditions. Among the most challenging reactions are measurements of $(\alpha, p)$ reaction studies and inelastic scattering in the active gas mode where multiple states are populated in the exit channel and energy is carried away by gamma rays. Results of a simulation of the ${ }^{14} \mathrm{O}(\alpha, \mathrm{p}){ }^{17} \mathrm{~F}$ are shown in Fig. 4 for a $25 \mathrm{MeV}$ beam of ${ }^{14} \mathrm{O}$ bombarding an extended $\mathrm{He}$ gas target of 120 Torr through a $2 \mu \mathrm{m}$ thick Ti foil. The $2 \mathrm{D}$ plot shows the reconstructed vertex (y axis) for ${ }^{14} \mathrm{O}(\alpha, \mathrm{p}){ }^{17} \mathrm{~F}_{\mathrm{gs}}$ and ${ }^{14} \mathrm{O}(\alpha, \mathrm{p}){ }^{17} \mathrm{~F}^{*}(495 \mathrm{keV})$ reactions producing protons with identical energy and hit location in the silicon strip array but originating from different locations in the gas (different center-of-mass energies). A center-of-mass energy resolution of a few hundred $\mathrm{keV}$ is typically expected in active gas target mode, limited by the emittance of the beam and straggling in the gas and windows.

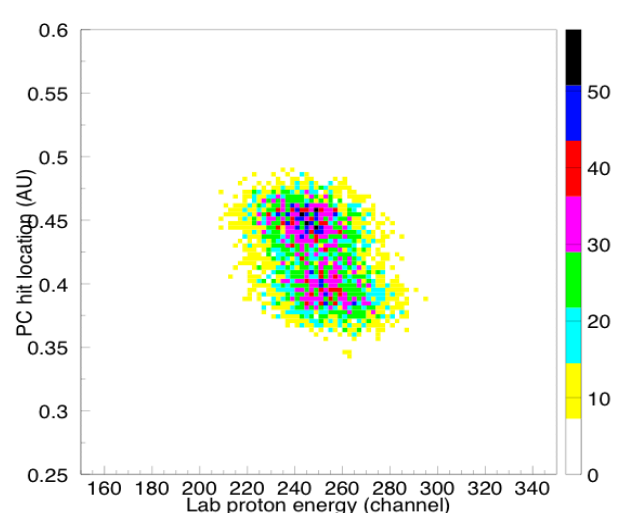

Figure 4. Results of a Monte Carlo simulation of the ${ }^{14} \mathrm{O}(\alpha, p)^{17} \mathrm{~F}$ reaction.

\section{Implementation}

ANASEN will be used primarily for experiments with radioactive ion beams at the RESOLUT facility at Florida State University (FSU) and at the ReA3 facility at the National Superconducting Cyclotron Laboratory (NSCL). Individual beamline components are being fabricated to accommodate ANASEN at both facilities, and the hardware at FSU where ANASEN will first be deployed is largely complete. Individual testing with sources of the prototypes for each detector element are now wrapping up, and testing with stable beams at FSU will begin in the near future.

The design of the beamline components for the NSCL implementation are now being completed, and will be fabricated and installed in 2011. A total of 5 letters of intent were submitted to and accepted by the NSCL Program Advisory Committee for experimental programs using ANASEN at ReA3 including measurements of $(\alpha, p)$ reactions, proton elastic and inelastic scattering, and transfer reaction studies such as $(\mathrm{d}, \mathrm{p})$ and $(\mathrm{d}, \mathrm{n})$. ANASEN will allow a broad range of experimental studies with high efficiency and sensitivity.

\section{References}

[1] S.D. Pain et al., Nucl. Instr. and Meth. Phys. Res. B 261 (2007) 1122.

[2] G.L. Engel et al., Nucl. Instr. and Meth. Phys. Res. A 573 (2007) 418.

[3] M.S. Wallace et al., Nucl. Instr. and Meth. Phys. Res. A 583 (2007) 302.

[4] B. Davin et al., Nucl. Instr. and Meth. Phys. Res. A 473 (2001) 302. 